\title{
ON THE VARIETY OF MANIFOLDS WITHOUT CONJUGATE POINTS $\left({ }^{1}\right)$
}

\author{
BY \\ ROBERT GULLIVER
}

\begin{abstract}
The longest geodesic segment in a convex ball of a riemannian manifold, where the convexity is ensured by an upper bound on sectional curvatures, is the diameter. This and related results are demonstrated and applied to show that there exist manifolds with sectional curvatures of both signs but without conjugate points.
\end{abstract}

1. Introduction. The central result of this paper is that no geodesic segment in a ball $B_{r_{0}}$ of a riemannian manifold has length greater than $2 r_{0}$, provided that the following property holds. The sectional curvatures at distance $r$ from the center are $\leqslant f(r)$, where $f$ is a monotone decreasing function on $\left[0, r_{0}\right]$. $f$ must be such that this curvature estimate implies that the concentric balls $B_{r}$ are convex for $r \leqslant r_{0}$ and that no geodesic through the center has focal points in $B_{r_{0}}$ along an orthogonal geodesic through the center. In particular, if $f$ is a positive constant $b^{2}$, then I require $b r_{0} \leqslant \pi / 2$. These results will be proved in $\S 2$.

The questions which stimulated this investigation, and the applications which will be discussed in $\S 3$ below, involve the existence problem for manifolds with curvatures of both signs whose geodesics have nonconjugacy or nonfocality properties. Nonconjugacy is the property that no nontrivial perpendicular Jacobi field along a geodesic vanishes at two points; equivalently, the exponential map from the tangent space of each point is nonsingular. Nonfocality is the stronger property that if any nontrivial perpendicular Jacobi field along any geodesic vanishes at some point, then its length has nonzero derivative at all other points; equivalently, the exponential map from the normal bundle of every geodesic $\tau$ is nonsingular (such a singular point would be a focal point of $\tau$ ). These properties are related to questions of divergence of geodesics and ergodicity of the geodesic flow. For example, the compact examples $A_{0}$ and $B_{0}^{2}$ constructed in $\S 3$ have Anosov geodesic flow (fọr definition see [7]); $B_{0}^{2}$ is a surface with focal points.

Received by the editors February 25, 1974 and, in revised form, July 20, 1974. AMS(MOS) subject classifications (1970). Primary 53C20; Secondary 58F15, 28A65.

Key words and phrases. Conjugate points, riemannian manifold, length of geodesic segments, Anosov geodesic flow.

(1) This research was partially supported by the United States Air Force Office of Scientific Reasearch under contract number AF-AFOSR 71-2098.

Copyright @ 1975. American Mathematical Society 
Klingenberg has recently shown that a manifold with Anosov geodesic flow must be free of conjugate points [7]; it is now shown that the manifold need not be free of focal points. Hypotheses previously known to imply nonconjugacy have required properties that were not, to my knowledge, shown to be satisfied on any manifold on which positive sectional curvatures occur.

It follows from the methods of this paper that, for example, the following condition excludes conjugacy on a riemannian manifold $M$ : the exponential map at some point embeds a ball of radius $r+R$ into $M$ such that all sectional curvatures on the closed annulus between $r$ and $r+R$ are $<-\beta^{2},<b^{2}$ inside the annulus and $<0$ beyond the annulus; where $b r<\pi / 2$ and $b \tan b r<\beta \tanh \beta R$. Previously known conditions have required specific knowledge of the geodesic paths, whereas the property of nonconjugacy is commonly required for detailed study of the geodesics, a somewhat unsatisfactory situation. It should be observed that the condition stated above is an open condition in that it is preserved under any $C_{0}^{2}$-small perturbation of the riemannian metric.

I am indebted to Pat Eberlein for posing questions which stimulated this investigation, and to Leon Green for several suggestions and references. I would also like to thank the referee for pointing out certain incomplete arguments and suggesting a number of improvements.

2. The growth of the distance function. In the first part of this section, I shall consider a riemannian manifold $M$ whose sectional curvatures, by way of normalization, are bounded above by 1 . Fix a point $p$ of $M$ and define the distance function $\rho(q)=d(p, q)$. Denote $B_{r}=\{q \in M: \rho(q)<r\}$. For some radius $r_{0}<\pi / 2$, assume that $B_{r_{0}}$ is the diffeomorphic image of the corresponding ball centered at the origin in the tangent space $M_{p}$ to $M$ at $p$ under the exponential map at $p . X=\operatorname{grad} \rho$ defines a smooth vector field on $B_{r_{0}}$ except at $p$. For $U \in$ $M_{q}, q \in B_{r_{0}}, q \neq p$, define

$$
k(U)=\left\langle\nabla_{U} X, U\right\rangle /\left(\|U\|^{2}-\langle X, U\rangle^{2}\right) .
$$

Then $k(U)$ is the normal curvature of the sphere $\partial B_{\rho(q)}$ with respect to the inward unit normal $-X$, in the direction given by the orthogonal projection of $U$ to the tangent space of the sphere. A brief computation yields

Lemma 1. For a unit-speed curve $\sigma$, define $\varphi(s)=\rho(\sigma(s))$. Then

$$
\varphi^{\prime \prime}=\left(1-\left(\varphi^{\prime}\right)^{2}\right) k\left(\sigma^{\prime}\right)+\left\langle X, \sigma^{\prime \prime}\right\rangle .
$$

In particular, if $\sigma$ is a geodesic, then

$$
\varphi^{\prime \prime}=\left(1-\left(\varphi^{\prime}\right)^{2}\right) k\left(\sigma^{\prime}\right) .
$$


Now an unintegrated form of the Rauch comparison theorem gives the estimate $k\left(\sigma^{\prime}(s)\right) \geqslant \cot \varphi(s)$ whenever $\varphi(s)<r_{0}$ (cf. [1, p. 251], or Lemma 3 below). This implies that $\varphi$ is a subsolution of the ordinary differential equation

$$
\psi^{\prime \prime}-\left(1-\left(\psi^{\prime}\right)^{2}\right) \cot \psi(s)=0,
$$

which is satisfied by the distance function along a geodesic in the sphere of radius 1 , as may be seen from (1). Given a constant $s_{0}$, a family of solutions $\psi_{t}$ to (2) may be defined by $\cos \psi_{t}(s)=\cos t \cos \left(s-s_{0}\right), 0 \leqslant \psi_{t}(s) \leqslant \pi$, according to the law of cosines of spherical trigonometry. Observe that $\psi_{t}(s) \geqslant\left|s-s_{0}\right|$ for $\mid s-$ $s_{0} \mid \leqslant \pi / 2$ and $|t|<\pi$.

Let $\sigma$ be a unit-speed geodesic on $M, \sigma\left(\left(s_{1}, s_{2}\right)\right)$ a segment of $\sigma$ which lies in $B_{r_{0}}$. I wish. to show $s_{2}-s_{1} \leqslant 2 r_{0}$. Without loss of generality, I may assume that $s_{1}$ and $s_{2}$ are extended real numbers such that no larger open segment of $\sigma$ lies in $B_{r_{0}}$. The assumption $r_{0}<\pi / 2$ implies $\varphi^{\prime \prime}(s) \geqslant 0$ for $s_{1}<s<s_{2}$, using equation (1) and the comparison estimate. Moreover $\varphi^{\prime \prime}(s)=0$ can occur for some $s$ only if $\left|\varphi^{\prime}(s)\right|=1$, in which case $\sigma$ must coincide with the geodesic through $p$, which implies $s_{2}-s_{1}=2 r_{0}$. Now assume that for some $s,\left|\varphi^{\prime}(s)\right|<1$. Then $\left|\varphi^{\prime}(s)\right|<1$ and $\varphi^{\prime \prime}(s)>0$ for all $s \in\left(s_{1}, s_{2}\right)$, so that $\varphi$ assumes a positive minimum $t_{0}<r_{0}$ at a unique point $s_{0} \in\left(s_{1}, s_{2}\right)$.

Thus $\varphi$ agrees up to first order with $\psi_{t_{0}}$ at $s_{0}$. Since $\varphi$ is a subsolution of (2), $\varphi(s) \geqslant \psi_{t_{0}}(s)$ must hold on the interval $\left|s-s_{0}\right| \leqslant \pi / 2, s_{1}<s<s_{2}$. In fact, one may introduce new coordinates $(s, t)$ for the domain $0<\rho+s<\pi, 0<\rho-$ $s<\pi$, defined by $\rho=\psi_{t}(s)$. Then $\rho=\varphi(s)$ may be described by $t=\gamma(s)$, and $\gamma$ satisfies a differential inequality of the form $\gamma^{\prime \prime}(s)+a(s) \gamma^{\prime}(s) \geqslant 0$. This inequality is equivalent to $\left(\alpha(s) \gamma^{\prime}(s)\right)^{\prime} \geqslant 0$, where $\alpha$ is a positive integrating factor. But $\gamma^{\prime}\left(s_{0}\right)=0$, so that $\gamma^{\prime}\left(s_{0}\right) \geqslant 0$ for $s \geqslant s_{0}$ and $\gamma^{\prime}(s) \leqslant 0$ for $s \leqslant s_{0}$. This implies $t=\gamma(s) \geqslant \gamma\left(s_{0}\right)=t_{0}$, which is to say $\varphi(s)=\psi_{t}(s) \geqslant \psi_{t_{0}}(s) \geqslant\left|s-s_{0}\right|$. In particular, if $s_{2}$ were greater than $s_{0}+r_{0}$, this would imply $\varphi\left(s_{0}+r_{0}\right) \geqslant$ $\psi_{t_{0}}\left(s_{0}+r_{0}\right) \geqslant r_{0}$, contradicting the assumption $\sigma\left(\left(s_{1}, s_{2}\right)\right) \subset B_{r_{0}}$. Thus $s_{2} \leqslant$ $s_{0}+r_{0}$, and by a similar argument $s_{1} \geqslant s_{0}-r_{0}$. This proves

THEOREM 1. Suppose $M$ is a riemannian manifold with all sectional curvatures $\leqslant b^{2}, B_{r_{0}}$ a ball in $M$ which is the diffeomorphic image under the exponential map of $a$ ball in the tangent space at its center. If $b r_{0}<\pi / 2$, then any geodesic segment in $B_{r_{0}}$ has length at most $2 r_{0}$.

The hypothesis regarding the magnitude of $r_{0}$ is precisely the bound required to ensure convexity of $B_{r}$ for $r \leqslant r_{0}$, or equivalently, to ensure that $p$ is not a focal point for any geodesic segment in $B_{r_{0}}$. One might be tempted to suppose that Theorem 1 should remain true if the curvature assumptions are replaced 
by the weaker convexity hypothesis. This seems unlikely. In any case, my methods of proof require some sort of a priori estimate on the growth of Jacobi fields along an arbitrary geodesic in $B_{r_{0}}$. However, the constant upper bound on curvature may be relaxed as in the theorem below. This strengthening is convenient, but not essential, for the examples constructed in $\S 3$. Observe that while the statement of Theorem 2 does not give sharp bounds on $r_{0}$, as in Theorem 1 , the sharp statement, in which $u_{1}$ and $u_{2}^{\prime}$ are required to be positive only on $\left[0, r_{0}\right)$, may be regained as a corollary. I denote $\rho(q)=d(p, q)$ as before.

THEOREM 2. Suppose $B_{r_{0}}$ is a ball in $M$ which is the diffeomorphic image under the exponential map of the corresponding ball in the tangent space at $p$. Assume that for all $q \in B_{r_{0}}$, all sectional curvatures $K$ for plane sections at $q$ satisfy $K \leqslant f(\rho(q))$, where $f$ is monotone decreasing on $\left[0, r_{0}\right]$. Suppose $f$ has the property that the solution $u_{1}$ of the Jacobi equation $u^{\prime \prime}+f u=0$ satisfying $u_{1}(0)=1, u_{1}^{\prime}(0)=0$ is positive on $\left[0, r_{0}\right]$, and the solution $u_{2}$ satisfying $u_{2}(0)=$ $0, u_{2}^{\prime}(0)=1$ has positive first derivative on $\left[0, r_{0}\right]$. Then any geodesic segment in $B_{r_{0}}$ has length at most $2 r_{0}$, and, moreover, cannot contain two mutually conjugate points.

Since I have not required $f$ to be continuous, a word is in order about the notion of solution to the Jacobi equation. When $f$ is of class $L_{1}$, I define this to mean a locally bounded solution to the corresponding integral equation:

$$
u_{1}(r)=1-\int_{0}^{r} \int_{0}^{t} f(s) u_{1}(s) d s d t
$$

and a similar equation for $u_{2}$, with $r$ as the first term. Solutions to these equations exist and are unique for $f \in L_{1}\left(\left[0, r_{0}\right]\right)$; moreover, the solutions $u_{1}$ and $u_{2}$, in the norm topology of $C^{1}\left(\left[0, r_{0}\right]\right)$, depend continuously on $f$ as $f$ varies in the norm topology of $L_{1}\left(\left[0, r_{0}\right]\right)$ (cf. [8, note Remark 2, p. 151]). In the interest of computational simplicity, one may observe that for piecewise continuous $f, u_{1}$ and $u_{2}$ are characterized as the $C^{1}$ functions which satisfy the Jacobi equation except at the discontinuities of $f$. Further, the integral-equation definition is equivalent to the notion of distributional solution:

$$
\int\left(u^{\prime} \varphi^{\prime}-f u \varphi\right)=0
$$

for every smooth function $\varphi$ with compact support in the domain of integration. The fact that a solution of the integral equation is a distributional solution will be used in $\S 3$, and may be verified by writing $u^{\prime}$ in terms of the integral equation and applying Fubini's theorem.

I claim that $f$ may be approximated from above in the $L_{1}$ norm by a func- 
tion $g$ which is monotone decreasing on $\left[0, r_{0}\right]$, and of class $C^{1}$ together with its even extension. Choose a small positive number $\epsilon$. Define $F(r)=f(|r|-\epsilon)$ for $|r| \geqslant \epsilon$ and $F(r)=f(0)$ for $|r| \leqslant \epsilon$. Then

$$
\|f-F\|_{L_{1}\left(\left[-r_{0}, r_{0}\right]\right)} \leqslant 2 \epsilon\left(f(0)-f\left(r_{0}\right)\right) .
$$

Now let $g$ be the convolution of $F$ with a positive, even $C^{1}$ kernel whose integral equals 1 and which vanishes outside of $(-\epsilon, \epsilon)$. Then

$$
\|g-F\|_{L_{1}\left(\left[-r_{0}, r_{0}\right]\right)} \leqslant 2 \epsilon\left(f(0)-f\left(r_{0}\right)\right) .
$$

Moreover, $g$ is of class $C^{\mathbf{1}}$, is even, decreases monotonically on $\left[0, r_{0}\right]$, and majorizes $f$.

Let $v_{1}, v_{2}$ be the solutions of the Jacobi equation $v^{\prime \prime}+g v=0$ with initial conditions $v_{1}(0)=v_{2}^{\prime}(0)=1, v_{1}^{\prime}(0)=v_{2}(0)=0$. As $\epsilon$ tends to zero, $v_{1}$ and $v_{2}^{\prime}$ tend uniformly to $u_{1}$ and $u_{2}^{\prime}$ on $\left[0, r_{0}\right]$. In particular, if $\epsilon$ is chosen small enough, then $v_{1}(r)>0$ and $v_{2}^{\prime}(r)>0$ for $0 \leqslant r \leqslant r_{0}$. Further, in the notation of the theorem, $K \leqslant g(\rho(q))$ since $g \geqslant f$. Therefore, without loss of generality, I may assume that $f$ is a class $C^{1}$, even function on $\left[-r_{0}, r_{0}\right]$.

Theorem 2 may now be proved in an analogous fashion to Theorem 1, by comparing $M$ with a manifold $M^{*}$ whose sectional curvatures at $q^{*}$ for plane sections tangent to the radial geodesic from $p^{*}$ are all equal to $f\left(d\left(p^{*}, q^{*}\right)\right)$, where $p^{*}$ is a fixed point of $M^{*}$. To construct $M^{*}$, define the first fundamental form in spherical normal coordinates centered at $p^{*}$ by $d s^{2}=d r^{2}+u_{2}(r)^{2} d \theta^{2}$, where $r$ is the radial coordinate and $d \theta^{2}$ is the first fundamental form on the unit sphere in euclidean space, pulled back under the central projection map. Let $B_{r}^{*}$ denote the open ball in $M^{*}$ of radius $r$ and center $p^{*}$. The crucial point which is needed regarding $M^{*}$ is that the family $\psi_{t}$ of solutions to the differential equation

$$
\psi_{t}^{\prime \prime}-\left(1-\left(\psi_{t}^{\prime}\right)^{2}\right) c\left(\psi_{t}\right)=0
$$

with initial conditions $\psi_{t}\left(s_{0}\right)=t, \psi_{t}^{\prime}\left(s_{0}\right)=0$, has the properties $(\partial / \partial t) \psi_{t}(s)>0$ and $\psi_{t}(s) \geqslant\left|s-s_{0}\right|$ for $\left|s-s_{0}\right|<r_{0}, 0<t<r_{0}$. Here $c(r)$ is the inward normal curvature of $\partial B_{r}^{*}$. These properties of the family $\psi_{t}(s)$ will be considered in Lemma 2 below. With the observation that $u_{2}$ is the length of a Jacobi field along a geodesic through $p^{*}$, whose Lie bracket with the unit radial vector field vanishes, a standard computation shows that $c=u_{2}^{\prime} / u_{2}$.

It follows from the unintegrated Rauch comparison theorem as before that under the hypotheses of Theorem $2, k\left(\sigma^{\prime}(s)\right) \geqslant c(\varphi(s))$ for any geodesic $\sigma$ in $B_{r_{0}}$, where $\varphi(s)=\rho(\sigma(s))$. Then according to Lemma $1, \varphi$ is a subsolution of equation (3) as long as $\sigma(s)$ is in $B_{r_{0}}$. As in the proof of Theorem 1, one may now 
show that $\varphi$ assumes a minimum at a unique $s_{0}$, and that $\varphi(s) \geqslant\left|s-s_{0}\right|$ as long as $\varphi(s)$ remains $<r_{0}$. In fact, if $\sigma$ passes through $p$, then $\varphi(s)=\left|s-s_{0}\right|$. Otherwise, we have $\varphi\left(s_{0}\right)=t_{0}>0$. Now using the results of Lemma 2, one sees that $\rho=\psi_{t}(s)$ defines a diffeomorphism between the domain $U_{1}=\left\{(s, \rho): 0<\rho<r_{0}\right.$, $\left.\left|s-s_{0}\right|<\rho\right\}$ and a neighborhood $U_{2}$ of the segment $s=s_{0}, 0<t<r_{0}$, in the $(s, t)$-plane. The equation $\rho=\varphi(s)$ with $\varphi\left(s_{0}\right)=t_{0}, \varphi^{\prime}\left(s_{0}\right)=0$ becomes $t=\gamma(s)$ with $\gamma\left(s_{0}\right)=t_{0}, \gamma^{\prime}\left(s_{0}\right)=0$. As long as the graph of $\gamma$ remains in $U_{2}$, an inequality of the form $\gamma^{\prime \prime}+a \gamma^{\prime} \geqslant 0$ holds, implying $\gamma(s) \geqslant t_{0}$ and $\varphi(s)=\psi_{t}(s) \geqslant$ $\psi_{t_{0}}(s)>\left|s-s_{0}\right|$ as before. In particular, the graph of $\varphi$ remains in $U_{1}$ (and the graph of $\gamma$ remains in $U_{2}$ ) as long as $\varphi(s)<r_{0}$. This implies $s_{2} \leqslant s_{0}+r_{0}$ and $s_{1} \geqslant s_{0}-r_{0}$, where $\sigma\left(\left(s_{1}, s_{2}\right)\right)$ is a maximal interval in $B_{r_{0}}$. Therefore $s_{2}-s_{1} \leqslant$ $2 r_{0}$, which gives the first conclusion of Theorem 2 .

To obtain the second conclusion, observe that the sectional curvatures at $\sigma(s)$ are $\leqslant f(\varphi(s)) \leqslant f\left(\left|s-s_{0}\right|\right)$. Now let $V$ be any nontrivial Jacobi field along $\sigma$, and suppose $V\left(s^{*}\right)=0$ for some $s^{*} \in\left[s_{1}, s_{2}\right]$. Let $u(s)$ be the unique solution of $u^{\prime \prime}(s)+f\left(\left|s-s_{0}\right|\right) u(s)=0$ satisfying $u\left(s^{*}\right)=0, u^{\prime}\left(s^{*}\right)=\left\|V^{\prime}\left(s^{*}\right)\right\|$. Then since $u_{1}\left(s-s_{0}\right)$ satisfies the same equation and is positive for $s_{1} \leqslant s \leqslant s_{2}$, it follows from the Sturm separation theorem that $s^{*}$ is the only zero of $u$ in $\left[s_{1}, s_{2}\right]$. Now $u(s)$ is the length of a Jacobi field in $M^{*}$ along a geodesic $\sigma^{*}$ with $\sigma^{*}\left(s_{0}\right)=p^{*}$. $V$ may be compared with this Jacobi field using the Rauch comparison theorem to conclude that $V(s) \neq 0$ for $s_{1} \leqslant s \leqslant s_{2}, s \neq s^{*}$ (see [1, p. 251] or Lemma 3 below).

It remains only to study the solutions of equation (3), that is, the growth of the distance function along geodesics of $M^{*}$. I may assume $s_{0}=0$ and that $M^{*}$ has dimension two, since only the autonomous equation (3) is being considered.

Lemma 2. Suppose for all $q^{*} \in B_{r_{0}}^{*}$ that the Gauss curvature of the surface $M^{*}$ at $q^{*}$ equals $f\left(d\left(p^{*}, q^{*}\right)\right)$, where $f$ is a smooth even function. For the solutions $u_{1}, u_{2}$ of $u^{\prime \prime}+f u=0$ satisfying $u_{1}(0)=u_{2}^{\prime}(0)=1, u_{1}^{\prime}(0)=u_{2}(0)=0$, assume that $u_{1}(r)>0, u_{2}^{\prime}(r)>0$ and $f^{\prime}(r) \leqslant 0$ for $0 \leqslant r \leqslant r_{0}$. Let $\tau$ be a unitspeed geodesic in $M^{*}, \tau(0)=p^{*}$. For $0<t<r_{0}$, let $\delta_{t}$ be the unit-speed geodesic with $\delta_{t}(0)=\tau(t), \delta_{t}^{\prime}(0)$ orthogonal to $\tau^{\prime}(t)$ and forming a continuous vector field along $\tau$. Denote

Then for each $t \in\left(0, r_{0}\right)$,

$$
\psi_{t}(s)=d\left(p^{*}, \delta_{t}(s)\right)
$$

(i) $(\partial / \partial t) \psi_{t}(s)>0$,

(ii) $\psi_{t}(s) \geqslant|s|$,

for all $s$ such that the open segment along $\delta_{t}$ from $\delta_{t}(0)$ to $\delta_{t}(s)$ lies in $B_{r_{0}}^{*}$.

Proof. Observe that $\psi_{t}$ is an even function, with $\left|\psi_{t}^{\prime}(s)\right| \leqslant 1$, which satis- 
fies equation (3) according to Lemma 1. For $0<t<r_{0}$, denote

$$
h(t)=\inf \left\{s>0: \delta_{t}(s) \notin B_{r_{0}}^{*}\right\} .
$$

I assert that for $t>0,\left|\psi_{t}^{\prime}(s)\right|<1$; for if $\psi_{t}^{\prime}(s)= \pm 1$, then $\psi_{t}^{\prime}$ is constant, as may be seen from the uniqueness theorem of ordinary differential equations, whereas $\psi_{t}^{\prime}(0)=0$ according to construction, a contradiction. Now $c$ is positive on $\left(0, r_{0}\right]$, so that equation (3) implies $\psi_{t}^{\prime \prime}(s)>0$ for all $s$ such that $\psi_{t}(s) \leqslant r_{0}$. Thus $\psi_{t}^{\prime}(s)$ has the same sign as $s$ for $|s| \leqslant h(t)$. Now $h(t)>0$ and satisfies $\psi_{t}(h(t))=r_{0}$. But $\psi_{t}^{\prime}(s)>0$ for $s>0$; therefore $h$ is differentiable and satisfies

$$
\psi_{t}^{\prime}(h(t)) h^{\prime}(t)=-\alpha(t, h(t))
$$

where $\alpha(t, s)$ denotes $(\partial / \partial t) \psi_{t}(s)$.

First, for some $t>0$, suppose that conclusion (ii) holds, that is, $\psi_{t}(s) \geqslant$ $|s|$ for $s \in[-h(t), h(t)]$. Then $\tau$ has no focal points along $\delta_{t}([-h(t), h(t)])$. For if $v(s)$ denotes the length of a nontrivial $\tau$-Jacobi field along $\delta_{t}$, then $v$ satisfies the Jacobi equation $v^{\prime \prime}(s)+f\left(\psi_{t}(s)\right) v(s)=0$ with the initial conditions $v^{\prime}(0)=0$, $v(0)>0$. Since $f\left(\psi_{t}(s)\right) \leqslant f(|s|)=f(s)$ by the monotonicity condition on $f, v(s)$ cannot vanish for $|s| \leqslant \inf \left\{r_{0}, h(t)\right\}$, according to the Sturm comparison theorem and the assumption that $u_{1}(r)>0$ on $\left[-r_{0}, r_{0}\right]$. Again using the supposition $\psi_{t}(s) \geqslant|s|$, one sees that $h(t)=|h(t)| \leqslant \psi_{t}(h(t))=r_{0}$, so that $v(s)>0$ for $|s| \leqslant$ $h(t)$ in particular. That is, $\tau$ has no focal points along $\delta_{t}$.

On the other hand, for some $t_{0}>0$, suppose that $\tau$ has no focal points along $\delta_{t}([-h(t), h(t)])$ for $0<t<t_{0}$. Then the $\tau$-Jacobi field $V(s)=(\partial / \partial t) \delta_{t}(s)$ along $\delta_{t}$ does not vanish for $|s| \leqslant h(t)$. Since $\alpha(t, 0)=(d / d t) d\left(p^{*}, \tau(t)\right)=1$, $\alpha(t, s)$ will remain positive unless $\alpha\left(t, s_{3}\right)=0$ for some $s_{3}, 0<\left|s_{3}\right| \leqslant h(t)$. But this would mean that $V\left(s_{3}\right)$ is orthogonal to the geodesic from $p^{*}$ to $\delta_{t}\left(s_{3}\right)$; since $M^{*}$ is two-dimensional, it would follow that this geodesic must coincide with $\delta_{t}$, an impossibility for $t>0$. Therefore $\alpha(t, s)>0$, which is the inequality of conclusion (i), for $0<t<t_{0},|s| \leqslant h(t)$. According to equation (4) this ensures that $h$ is decreasing on $0<t<t_{0}$, so that $\alpha(t, s)>0$ holds for $0<t<t_{0}$, $|s| \leqslant h\left(t_{0}\right)$. With the observation that $\psi_{t}(s) \rightarrow|s|$ as $t \rightarrow 0$, one may integrate with respect to $t$ to show that $\psi_{t_{0}}(s)>|s|$ for $|s| \leqslant h\left(t_{0}\right)$. From this it follows that for some $\epsilon>0, \psi_{t}(s)>|s|$ for all $t \in\left[t_{0}, t_{0}+\epsilon\right)$ and $|s| \leqslant h(t)$. As shown in the preceding paragraph, this implies that $\tau$ has no focal points along $\delta_{t}([-h(t), h(t)])$ for $0<t<t_{0}+\epsilon$.

Finally let $t_{0}$ be the infimum of all $t>0$ such that $\tau$ has focal points along $\delta_{t}([-h(t), h(t)])$. Since $\psi_{0}(s)=|s|$, there are no focal points of $\tau$ along $\delta_{0}\left(\left[-r_{0}, r_{0}\right]\right)$, as shown above. Using the continuity of $h$ and the fact that focal 
points correspond to a closed subset of the normal bundle of $\tau$, one may verify that $t_{0}>0$. The above argument shows that if $t_{0}<r_{0}$, then there exists $\epsilon>0$ such that $\tau$ has no focal points along $\delta_{t}([-h(t), h(t)])$ for $0<t<t_{0}+\epsilon$; this contradicts the choice of $t_{0}$. Therefore $\tau$ has no focal points along $\delta_{t}([-h(t), h(t)])$ for all $t \in\left[0, r_{0}\right)$. Conclusions (i) and (ii) now follow from the above proof.

3. Construction of manifolds without conjugate points. With the results of the previous section, it is now possible to construct explicitly complete manifolds with curvature of both signs, whose geodesics exhibit various aspects of behavior typical of manifolds with negative curvature. The examples themselves are not surprising, and their construction is widely understood.

Four specific classes of manifolds may be singled out, for which examples will be given. Examples $A$ and $A_{0}$ : no focal points occur (and hence no conjugate points), dimension $\geqslant 2$ is arbitrary; $A_{0}$ is compact, $A$ is noncompact. Examples $B$ and $B_{0}^{2}$ : focal points occur, but no conjugate points; $B_{0}^{2}$ is compact and two-dimensional, $B$ is noncompact and has arbitrary dimension. Each compact example has Anosov geodesic flow. The topological type of $A_{0}$ or $B_{0}^{2}$ may be chosen as that of any manifold or orientable surface, respectively, which carries a metric of constant negative sectional curvature. Each example may be made real-analytic. Nonexistence of conjugate points implies that the universal covering manifold is diffeomorphic to euclidean space. From this follows, for example, that the existence of $A_{0}$ implies existence for $A$. $A$ and $B$, however, admit direct constructions, while $A_{0}$ and $B_{0}^{2}$ are formed by "raising a blister" on compact manifolds of constant negative curvature.

Let the first fundamental form of an $n$-dimensional riemannian manifold $M$ be given in geodesic polar coordinates from a point $p$ of $M$ by

$$
d s^{2}=d r^{2}+G(r)^{2} d \theta^{2},
$$

where $d \theta^{2}$ is the pullback under radial projection of the fundamental form of the unit $(n-1)$-sphere in euclidean space. $G$ satisfies the Jacobi equation

$$
G^{\prime \prime}+K_{1} G=0
$$

where $K_{1}(r)$ is the sectional curvature of any plane section containing the radial direction at any point at distance $r$ from $p$. $G$ satisfies initial conditions $G(0)=$ $0, G^{\prime}(0)=1$. Let $\lambda(r)=G^{\prime}(r) / G(r)$. Then $\lambda$ satisfies the Riccati equation

$$
\lambda^{\prime}+\lambda^{2}+K_{1}=0
$$

with the initial condition that $\lambda(r)-r^{-1} \rightarrow 0$ as $r \rightarrow 0$. I shall use equation 
(5) or (6) to determine $K_{1}$. G may be regained from $\lambda$ by integrating:

$$
\log G(r)=\log r+\int_{0}^{r}\left(\lambda(t)-t^{-1}\right) d t .
$$

Observe that $G$ and $\lambda$ are analogous to the functions $u_{2}$ and $c$ of Lemma 2. Let $K_{2}(r)$ denote the sectional curvature of any plane section orthogonal to the radial direction at distance $r$ from $p$. Then $K_{2}=G^{-2}-\lambda^{2} . K_{1}$ and $K_{2}$ represent the extreme values of sectional curvature in a metric of this form. In fact, the sectional curvature corresponding to a plane section which makes an angle $\theta$ with the radial direction is $[2$, p. 27]

$$
K=K_{1} \cos ^{2} \theta+K_{2} \sin ^{2} \theta .
$$

I shall define $\lambda$ and $G$ as approximations to functions $\lambda_{0}$ and $G_{0}$, where $\lambda_{0}$ and $G_{0}^{\prime}$ are discontinuous. Choose positive constants $b$ and $r_{1}$ with $b r_{1}<\pi / 2$. For $0<r<r_{1}$, set $\lambda_{0}=b \cot b r$; the resulting sectional curvatures are all $=b^{2}$. There exists a unique $r_{2}, 0<r_{2}<r_{1}$, so that $b^{-1} \sin b r_{1}=\sinh \left(r_{1}-r_{2}\right)$. Define $\lambda_{0}(r)=\operatorname{coth}\left(r-r_{2}\right)$ for $r>r_{1}$; the resulting sectional curvatures for plane sections tangent to the radial direction are $K_{1}=-\lambda_{0}^{\prime}-\lambda_{0}^{2}=-1$. The relation defining $r_{2}$ implies that $G_{0}(r)=\sinh \left(r-r_{2}\right)$ for $r>r_{1}$. Thus $K_{2}=G_{0}^{-2}-$ $\lambda_{0}^{2}=-1$, as well. Now for some small positive $\epsilon<r_{1}-r_{2}$, modify $\lambda_{0}$ on $\left(r_{1}-\right.$ $\left.\epsilon, r_{1}+\epsilon\right)$ to yield a function $\lambda$ of class $C^{1}$ whose integral on $\left(r_{1}-\epsilon, r_{1}+\epsilon\right)$ agrees with that of $\lambda_{0}$, such that $\lambda^{\prime}+\lambda^{2} \geqslant-b^{2}$ and $\lambda(r) \geqslant b \cot b r$. This is possible since $\operatorname{coth}\left(r-r_{2}\right)>\operatorname{coth} r>b \cot b r$ for $r>r_{2}$. More precisely, one may transform the equation $x=\lambda(r)$ into $\rho=k(r)$, where the relation $x=$ $b \cot b(r-\rho)$ defines a diffeomorphism of the $(r, x)$-plane with the strip $0<r-$ $\rho<\pi / b$ in the $(r, \rho)$-plane. Then the conditions $\lambda(r) \geqslant b \cot b r$ and $\lambda^{\prime}+\lambda^{2} \geqslant$ $-b^{2}$ become $k(r) \geqslant 0$ and $k^{\prime}(r) \geqslant 0$, both of which are satisfied by the function $k_{3}$ defined by $\operatorname{coth}\left(r-r_{2}\right)=b \cot b\left(r-k_{3}(r)\right), r>r_{2}$. Thus one may choose a smooth one-parameter family $k_{\alpha}, 1 \leqslant \alpha \leqslant 2$, such that for all $\alpha, k_{\alpha} \geqslant 0, k_{\alpha}^{\prime} \geqslant 0$; $k_{\alpha}(r)=0$ for $0 \leqslant r \leqslant r_{1}-\epsilon$; and $k_{\alpha}(r)=k_{3}(r)$ for $r \geqslant r_{1}+\epsilon$. I further ask that $k_{1}$ satisfy $k_{1}(r)=0$ on all of $\left[0, r_{1}\right]$, with $k_{1}(r) \leqslant k_{3}(r)$ for all $r>r_{2}$; and that $k_{2}$ satisfy $k_{2}(r)=k_{3}(r)$ for all $r \geqslant r_{1}$. Then the integral of the corresponding functions $\lambda_{\alpha}$ will vary continuously with $\alpha$, being less than the integral of $\lambda_{0}$ at $\alpha=1$ but greater at $\alpha=2$, so that for some intermediate value of $\alpha, \lambda=\lambda_{\alpha}$ has the same integral as $\lambda_{0}$. Observe that $G(r) \geqslant b^{-1} \sin b r$; thus the resulting bounds on sectional curvatures $K_{1}=-\lambda^{\prime}-\lambda^{2} \leqslant b^{2}$ and $K_{2}=G^{-2}-\lambda^{2} \leqslant b^{2}$ on $\left(r_{1}-\epsilon, r_{1}+\epsilon\right)$. The condition that $\lambda$ and $\lambda_{0}$ have the same integral implies that $G(r)=G_{0}(r)$ for $r \geqslant r_{1}+\epsilon$, from which $K_{2}=-1$ follows.

Choose $b, r_{1}$ and $\epsilon$ so that $b \tan b\left(r_{1}+\epsilon\right)<1$, with $b\left(r_{1}+\epsilon\right)<\pi / 2$. 
Then I claim that $M$ satisfies the hypotheses of Theorem 2 with any positive value for $r_{0}$, and where $f$ is the constant $b^{2}$ on the interval $\left[0, r_{1}+\epsilon\right]$ and equals -1 for all arguments greater than $r_{1}+\epsilon$. Writing $r_{3}=r_{1}+\epsilon$, one may compute explicitly $u_{1}(r)=\cos b r, u_{2}(r)=b^{-1} \sin b r$ for $0 \leqslant r \leqslant r_{3}$; and $u_{1}(r)=$ $\cos b r_{3} \cosh \left(r-r_{3}\right)-b \sin b r_{3} \sinh \left(r-r_{3}\right), u_{2}(r)=b^{-1} \sin b r_{3} \cosh \left(r-r_{3}\right)+$ $\cos b r_{3} \sinh \left(r-r_{3}\right)$ for $r \geqslant r_{3}$. Thus the hypotheses $u_{1}(r)>0$ and $u_{2}^{\prime}(r)>0$ follow from $b \tan b r_{3}<1$ and $b r_{3}<\pi / 2$.

For the construction of example $B$, I further require that $b\left(r_{1}-\epsilon\right)>\pi / 4$ (this forces $b<1$ ). The disk of radius $r_{1}-\epsilon$ is then isometric to a spherical cap which contains a segment of a great circle of length greater than one-fourth the circumference, so that either endpoint has a focal point along the segment. However, conjugate points are excluded by Theorem 2 .

Before considering example $A$, I shall give a comparison lemma which will be useful here and in the sequel. Although the result is valid in any number of dimensions, its proof is more closely related to methods of Sturm than to the Rauch comparison theorem.

Lemma 3. Let $\sigma$ be a geodesic in a riemannian manifold $M, V$ a perpendicular Jacobi field along $\sigma$, with length $v$. Suppose $f: \mathbf{R} \rightarrow \mathbf{R}$ is integrable on bounded sets, such that for all $s, K\left(\sigma^{\prime}(s), V(s)\right) \leqslant f(s)$. Choose $s^{*}$, and let $u$ be a distributional solution of $u^{\prime \prime}+f u=0$ with $u\left(s^{*}\right)=v\left(s^{*}\right), u^{\prime}\left(s^{*}\right) \leqslant v^{\prime}\left(s^{*}\right)$, and suppose $u(s)>0$ for $s^{*}<s \leqslant s^{* *}$. Then for $s^{*}<s \leqslant s^{* *}, v(s) \geqslant u(s)$ and

$$
v^{\prime}(s) / v(s) \geqslant u^{\prime}(s) / u(s)
$$

Proof. Since $V$ is a Jacobi field, its covariant derivatives $V^{\prime}$ and $V^{\prime \prime}$ along $\sigma$ satisfy $\left\langle V^{\prime \prime}, V\right\rangle+K\left(\sigma^{\prime}, V\right) v^{2}=0$, hence

$$
v^{\prime \prime}+f v \geqslant v^{\prime \prime}+K\left(\sigma^{\prime}, V\right) v=v^{-3}\left(\langle V, V\rangle\left\langle V^{\prime}, V^{\prime}\right\rangle-\left\langle V, V^{\prime}\right\rangle^{2}\right) \geqslant 0 .
$$

Note that $u$ is continuously differentiable. I shall show that the continuous function $\Delta=u v^{\prime}-v u^{\prime}$ is monotone increasing on $\left[s^{*}, s^{* *}\right]$. To this end, I shall first show that $\Delta$ has a nonnegative distributional first derivative. Let $\varphi$ be any nonnegative $C^{1}$ function with support in $\left(s^{*}, s^{* *}\right)$. Since $v$ satisfies $v^{\prime \prime}+f v \geqslant 0$, and since $u \geqslant 0$, integration by parts yields $\int\left(v^{\prime}(\varphi u)^{\prime}-f v(\varphi u)\right) \leqslant 0$ (this and the next two integrals are over $\left.\left[s^{*}, s^{* *}\right]\right)$. Meanwhile, $u$ is a distributional solution to $u^{\prime \prime}+f u=0$, so that $\int\left(u^{\prime}(\varphi v)^{\prime}-f u(\varphi v)\right)=0$. Subtraction of these integrals yields $\int \varphi^{\prime} \Delta \leqslant 0$, which expresses the fact that $\Delta$ has a nonnegative distributional first derivative. But this implies that $\Delta$ is monotone increasing: otherwise, I have $\Delta\left(s_{1}\right)>\Delta\left(s_{2}\right)$, where $s^{*} \leqslant s_{1}<s_{2} \leqslant s^{* *}$. By continuity, there exist $\delta>0$ and $\Delta_{0}$ such that for all $t_{1} \in\left[s_{1}, s_{1}+\delta\right)$ and $t_{2} \in\left(s_{2}-\delta, s_{2}\right]$ there holds $\Delta\left(t_{1}\right)>$ 
$\Delta_{0}>\Delta\left(t_{2}\right)$. Choose $\varphi$ to be a $C^{1}$ function with support in $\left(s_{1}, s_{2}\right)$, such that $\varphi^{\prime}>0$ on $\left(s_{1}, s_{1}+\delta\right), \varphi^{\prime}<0$ on $\left(s_{2}-\delta, s_{2}\right)$, and $\varphi^{\prime}=0$ otherwise. Then

$$
\int_{s_{1}}^{s_{1}+\delta} \varphi^{\prime} \Delta>\Delta_{0} \int_{s_{1}}^{s_{1}+\delta} \varphi^{\prime}=-\Delta_{0} \int_{s_{2}-\delta}^{s_{2}} \varphi^{\prime}>-\int_{s_{2}-\delta}^{s_{2}} \varphi^{\prime} \Delta
$$

and hence the integral of $\varphi^{\prime} \Delta$ over $\left[s^{*}, s^{* *}\right]$ is positive, a contradiction. Therefore, $\Delta$ is monotone increasing.

Now by hypothesis, $\Delta\left(s^{*}\right) \geqslant 0$, so that $\Delta(s) \geqslant 0$ for $s^{*} \leqslant s \leqslant s^{* *}$. This implies that $(v / u)^{\prime} \geqslant 0$, while $v(s) / u(s)$ tends to a limit $\geqslant 1$ as $s$ approaches $s^{*}$ from above (use L'Hôpital's rule in case $u\left(s^{*}\right)=0$ ). Therefore $v(s) \geqslant u(s)$ for all $s \in$ $\left(s^{*}, s^{* *}\right.$ ], as claimed. In particular, $v(s)>0$, so that inequality (7) follows from $\Delta(s) \geqslant 0$. This completes the proof of Lemma 3 .

I now return to the construction of examples. For example $A, r_{1}$ and $\epsilon$ need to be small enough that $b \tan 2 b r_{3}<1$ (here $r_{3}=r_{1}+\epsilon$ ). Let $\sigma$ be a geodesic in $M$. According to Theorem 2 with $r_{0}=r_{3}$, any segment of $\sigma$ which lies in $B_{r_{3}}$ has length at most $2 r_{3}$. Moreover, according to the proof of Theorem 2 with large $r_{0}$, the distance from $p$ to $\sigma(s)$ is a convex function of $s$; thus at most one segment of $\sigma$ lies in $B_{r_{3}}$. Therefore all sectional curvatures at $\sigma(s)$ are $\leqslant b^{2}$ and $=-1$ except for $s$ in an interval $\left(s_{1}, s_{2}\right)$ of length at most $2 r_{3}$. Let $v$ be the length of a perpendicular Jacobi field along $\sigma, v\left(s^{*}\right)=0$. In order to eliminate focal points, I shall show that $v^{\prime}(s)>0$ for all $s \geqslant s^{*}$. According to Lemma 3, and using the linearity of the Jacobi equation, this will follow if it can be shown that $u^{\prime}(s)>0$ for $s \geqslant s^{*}$, where $u$ is the distributional solution of $u^{\prime \prime}+f u=0$ satisfying $u\left(s^{*}\right)=0, u^{\prime}\left(s^{*}\right)=1$; and where $f(s)=b^{2}$ for $s_{1} \leqslant s \leqslant s_{2}, f(s)=-1$ otherwise. First consider the case $s^{*}<s_{1}$ : then $u(s)=\sinh \left(s-s^{*}\right)$ for $s^{*} \leqslant$ $s \leqslant s_{1}$. Then on $\left[s_{1}, s_{2}\right], u(s)=\sinh \left(s_{1}-s^{*}\right) \cos b\left(s-s_{1}\right)+\cosh \left(s_{1}-s^{*}\right) \cdot$ $b^{-1} \sin b\left(s-s_{1}\right)$, so that $u^{\prime}(s)>0$, since $b \tan b\left(s-s_{1}\right)<1$. Proceeding, one finds that for $s>s_{2}, u(s)=u\left(s_{2}\right) \cosh \left(s-s_{2}\right)+u^{\prime}\left(s_{2}\right) \sinh \left(s-s_{2}\right)$, so that $u^{\prime}(s)$ is again positive. The cases $s_{1} \leqslant s^{*}<s_{2}$ and $s_{2} \leqslant s^{*}$ may be handled similarly. This shows that $A$ is free of focal points.

I now turn to the compact examples. Suppose given a compact manifold $M^{\prime}$ of nonpositive sectional curvature, and a point $p^{\prime}$ of $M^{\prime}$ such that the exponential map at $p^{\prime}$ is injective on a ball of radius $R>r_{3}-r_{2}$. Assume that all sectional curvatures of $M^{\prime}$ on the ball of radius $R$ centered at $p^{\prime}$ equal -1 . The annulus $Q$ centered at $p^{\prime}$ with inner radius $r_{3}-r_{2}$ and outer radius $R$ may be mapped isometrically onto the annulus in $M$ (as constructed for example $A$ or $B$ ) with inner radius $r_{3}$ and outer radius $R+r_{2}$, by adding $r_{2}$ to the radial coordinate in a system of geodesic polar coordinates at $p^{\prime}$. Identifying these annuli, one 
may form a compact manifold $M$, in which the ball of radius $R$ centered at $p^{\prime}$ in $M^{\prime}$ is replaced by the ball of radius $R+r_{2}$ centered at $p$ in $M$. Let $D$ denote the smaller ball, of radius $r_{3}$, and retain the notation $Q$ for the annulus. Now let $\sigma$ be any geodesic in $M$. Any segment of $\sigma$ in $D$ has length at most $2 r_{3}$ according to Theorem 1. As in the proof of Theorem 2, the distance from $p$ to $\sigma(s)$ is a convex function of $s$ as long as $\sigma$ remains in $D \cup Q$. Thus, after $\sigma$ leaves $D$, it must cross $Q$ to its outer boundary, traveling a distance of at least $R^{\prime}=R+$ $r_{2}-r_{3}$. In summary, the sectional curvatures at $\sigma(s)$ are at most $b^{2}$ for $s$ in certain intervals of length less than $2 r_{3}$; these intervals are separated by intervals on which the sectional curvatures are nonpositive, with subintervals at each end of length $\geqslant R^{\prime}$ on which all sectional curvatures are -1 .

I shall show that conjugate points do not occur on $M$, provided that

$$
b \tan b r_{3}<\tanh R^{\prime} .
$$

Note that $b r_{3}<\pi / 2$ is still required. Let $\left[s_{k}, t_{k}\right]$ denote intervals such that sectional curvatures at $\sigma(s)$ are $=-1$ for $t_{k} \leqslant s \leqslant t_{k}+R^{\prime}$ and for $s_{k}-R^{\prime} \leqslant s \leqslant s_{k}$, $\leqslant b^{2}$ for $s_{k}<s<t_{k}$, and $\leqslant 0$ otherwise. I have $t_{k}-s_{k} \leqslant 2 r_{3}$ and $s_{k+1}-$ $t_{k} \geqslant 2 R^{\prime}$. I assume that $k$ ranges over an interval of the integers, $k_{1} \leqslant k \leqslant k_{2}$ (with the understanding that equality is excluded if $k_{1}$ or $k_{2}$ is infinite). Now let $v(s)$ be the length of a perpendicular Jacobi field along $\sigma, v\left(s^{*}\right)=0$; I shall show that $v$ vanishes only at $s^{*}$. According to Lemma 3 , it is enough to show that the solution $u$ of $u^{\prime \prime}+f u=0$ satisfying $u\left(s^{*}\right)=0, u^{\prime}\left(s^{*}\right)=1$, vanishes only at $s^{*}$; where $f(s)=b^{2}$ for $s_{k}<s<t_{k}, f(s)=-1$ for $t_{k} \leqslant s \leqslant t_{k}+R^{\prime}$ and for $s_{k}-R^{\prime} \leqslant s \leqslant s_{k}$, and $f(s)=0$ otherwise. By symmetry, I need only show $u(s)>0$ for $s>s^{*}$. Denote $\mu=u^{\prime} / u$. If $u\left(s^{* *}\right)$ were zero, then $\mu(s)$ would tend to $-\infty$ as $s$ approaches $s^{* *}$ from below. Thus it will be enough to bound $\mu$ from below for $s>s^{*}$.

I shall first establish the following sequence of statements:

(a) if $\mu\left(s_{k}-R^{\prime}\right) \geqslant 0$ then $\mu\left(s_{k}\right) \geqslant \tanh R^{\prime}>b \tan b r_{3}$;

(b) if $\mu\left(s_{k}\right)>b \tan b r_{3}$ then $\mu\left(t_{k}\right)>-b \tan b r_{3}>-\tanh R^{\prime}$;

(c) if $\mu\left(t_{k}\right)>-\tanh R^{\prime}$ then $\mu\left(t_{k}+R^{\prime}\right)>0$; and

(d) if $\mu\left(t_{k}+R^{\prime}\right) \geqslant 0$ then $\mu\left(s_{k+1}-R^{\prime}\right) \geqslant 0$.

These may be demonstrated by explicit computation of $\mu$. On the interval $\left[s_{k}-R^{\prime}, s_{k}\right]$, if $\left|\mu\left(s_{k}-R^{\prime}\right)\right|<1$ then $\mu(s)=\tanh \left(s-z_{0}\right)$ where $z_{0}$ is determined by $\mu\left(s_{k}-R^{\prime}\right)$. If $\mu\left(s_{k}-R^{\prime}\right) \geqslant 0$, then $z_{0} \leqslant s_{k}-R^{\prime}$; therefore $\mu\left(s_{k}\right) \geqslant \tanh R^{\prime}$, which is statement (a). If $\mu\left(s_{k}-R^{\prime}\right)=1$, then $\mu(s)=1$, and if $\mu\left(s_{k}-R^{\prime}\right)>1$, then $\mu(s)=\operatorname{coth}\left(s-s_{0}\right)>1$; statement (a) follows since $\tanh R^{\prime}<1$. Statement (c) is verified in a similar fashion. On $\left[s_{k}, t_{k}\right], \mu$ takes the form $\mu(s)=$ $b \cot b\left(s-z_{1}\right)$. If $\mu\left(s_{k}\right)>b \tan b r_{3}$ then $z_{1}>s_{k}+r_{3}-\pi / 2 b \geqslant t_{k}-r_{3}-\pi / 2 b$, 
and $\mu\left(t_{k}\right)>-b \tan b r_{3}$ follows, which is statement $(b)$. On $\left[t_{k}+R^{\prime}, s_{k+1}-R^{\prime}\right]$, $\mu$ is either identically zero or takes the form $\mu(s)=\left(s-z_{2}\right)^{-1}$; statement (d) follows readily.

Observe that by the same argument, in the case that $k_{2}$ is finite, if $\mu\left(t_{k_{2}}+R^{\prime}\right) \geqslant 0$ then $\mu(s) \geqslant 0$ for all $s \geqslant t_{k_{2}}+R^{\prime}$.

Now suppose $s^{*} \in\left[s_{n}, t_{n}\right]$ : then for $s_{n} \leqslant s \leqslant t_{n}, \mu(s)=b \cot b\left(s-s^{*}\right)$, so that $\mu\left(t_{n}\right) \geqslant b \cot 2 b r_{3}>-b \tan b r_{3}>-\tanh R^{\prime}$, since $b r_{3}<\pi / 2$. It follows by induction that statements (a)-(d) hold for all relevant values of $k \geqslant n$, and from the proof of these statements that $\mu(s)$ is bounded below for $s^{*}<s<$ $t_{k_{2}}+R^{\prime}$. But in particular, in the case that $k_{2}$ is finite, $\mu\left(t_{k_{2}}+R^{\prime}\right)>0$, from which it follows that $\mu(s) \geqslant 0$ for $s \geqslant t_{k_{2}}+R^{\prime}$. Therefore, in either case, $\mu$ is bounded from below.

If $s^{*} \in\left[t_{n}, t_{n}+R^{\prime}\right]$, then $\mu(s)=\operatorname{coth}\left(s-s^{*}\right)$ for $t_{n} \leqslant s \leqslant t_{n}+R^{\prime}$ and in particular $\mu\left(t_{n}+R^{\prime}\right)>0$. If $s^{*} \in\left[t_{n}+R^{\prime}, s_{n+1}-R^{\prime}\right]$, then $\mu(s)=\left(s-s^{*}\right)^{-1}$ for $t_{n}+R^{\prime} \leqslant s \leqslant s_{n+1}-R^{\prime}$, so that $\mu\left(s_{n+1}-R^{\prime}\right)>0$. If $s^{*} \in\left[s_{n}-R^{\prime}, s_{n}\right]$ then $\mu(s)=\operatorname{coth}\left(s-s^{*}\right)$ for $s_{n}-R^{\prime} \leqslant s \leqslant s_{n}$, and in particular $\mu\left(s_{n}\right)>1>$ $\tanh R^{\prime}$. In each case, the induction argument is started to show that $\mu$ is bounded from below. This shows that $M$ is free of conjugate points.

A further question of interest regarding $M$ is whether the geodesic flow satisfies the Anosov axioms. Eberlein has shown that in a compact manifold (or a riemannian covering of a compact manifold) without conjugate points, the Anosov property is equivalent to the unboundedness of every nonvanishing perpendicular Jacobi field $V$ along every geodesic $\sigma$ in $M$ [5, I]. I shall show that, in fact, every nontrivial perpendicular Jacobi field is unbounded; assuming still that $M$ is compact and inequality (8) holds, but requiring in addition that all sectional curvatures outside $B_{r_{3}}$ are $\leqslant-c^{2}<0$. Let $v$ denote the length of $V$ as before. Choose $x_{0}$ which is not in any of the intervals $\left(s_{k}-R^{\prime}, t_{k}+R^{\prime}\right)$, and for which $v\left(x_{0}\right) \neq 0$. I may assume $v^{\prime}\left(x_{0}\right) \geqslant 0$, since otherwise this will obtain if $s$ is replaced by $-s$. Let $u$ be a comparison function, the solution of $u^{\prime \prime}+f u=0$ with $u\left(x_{0}\right)=1$, $u^{\prime}\left(x_{0}\right)=0$, where $f$ is as before except that $f=-c^{2}$ on the complement of the intervals $\left(s_{k}-R^{\prime}, t_{k}+R^{\prime}\right)$. I may apply Lemma 3 to $v$ and an appropriate multiple of $u$ to show that if $u$ is unbounded, then $v$ is unbounded. Write $\mu=u^{\prime} / u$. I shall show that any indefinite integral of $\mu$, such as $\log u$, is unbounded; from this it follows that $u$ and $v$ are unbounded.

Now if $f=-c^{2}$ on some interval $(y, \infty)$ and $\mu(y) \geqslant 0$, then $\mu(s) \geqslant$ $c \tanh c(s-y)$. It follows that $\mu$ has a positive lower bound on $(y+1, \infty)$ so that its indefinite integral is unbounded. If $x_{0} \geqslant t_{k_{2}}+R^{\prime}$, or if there are no intervals $\left[s_{k}, t_{k}\right]$ at all, then this argument applies to the interval $\left(x_{0}, \infty\right)$ to yield the desired conclusion. Thus I may assume $x_{0}<s_{k_{2}}-R^{\prime}$. Also, if $k_{2}$ is finite, 
then I only need to show $\mu\left(t_{k_{2}}+R^{\prime}\right) \geqslant 0$ to reach the conclusion.

Let $\left[s_{n}, t_{n}\right]$ denote the first interval to the right of $x_{0}$. Since $\mu\left(x_{0}\right)=0$, $\mu\left(s_{n}-R^{\prime}\right)=c \tanh c\left(s_{n}-R^{\prime}-x_{0}\right)>0$. This starts the induction argument to show that statements (a)-(d) above all hold for $k \geqslant n$. In particular, if $k_{2}$ is finite, then $\mu\left(t_{k_{2}}+R^{\prime}\right) \geqslant 0$, and the conclusion follows. Thus I shall assume hereafter that $k_{2}$ is infinite.

On each interval $\left[s_{k}, t_{k}\right], \mu$ takes the form $\mu(s)=b \cot b\left(s-z_{1}\right)$; for $k \geqslant n$, $z_{1}>s_{k}+r_{3}-\pi / 2 b$ as in the proof of statement (b). In particular, $z_{1}+\pi / 2 b$ is to the right of the midpoint of $\left[s_{k}, t_{k}\right]$, since $t_{k}-s_{k} \geqslant 2 r_{3}$, which implies $\int_{s_{k}}^{t_{k}} \mu(s) d s>0$. Between $s_{k}-R^{\prime}$ and $s_{k}$, either $\mu$ has the form $\mu(s)=\tanh \left(s-z_{0}\right)$, where for $k \geqslant n, z_{0} \leqslant s_{k}-R^{\prime}$; or $\mu \geqslant 1$. In either case,

$$
\int_{s_{k}-R^{\prime}}^{s_{k}} \mu(s) d s>\int_{0}^{R^{\prime}} \tanh r d r .
$$

Between $t_{k}$ and $t_{k}+R^{\prime}$, either $\mu$ has the form $\mu(s)=\tanh \left(s-z_{3}\right)$, or $\mu \geqslant 1$. Define $z^{*}>-R^{\prime}$ so that $\tanh z^{*}=-b \tan b r_{3}$. If $k \geqslant n$ and $t_{k} \leqslant s \leqslant t_{k}+R^{\prime}$, then $\mu(s) \geqslant \tanh \left(z^{*}-t_{k}+s\right)$. Therefore

$$
\int_{t_{k}}^{t_{k}+R^{\prime}} \mu(s) d s \geqslant \int_{z^{*}}^{z^{*}+R^{\prime}} \tanh r d r=\int_{-R^{\prime}}^{0} \tanh r d r+\delta
$$

where $\delta>0$ is independent of $k$. Finally, $\mu \geqslant 0$ on the intervals $\left(t_{k}+R^{\prime}\right.$, $\left.s_{k+1}-R^{\prime}\right), k \geqslant n$. Adding these integral estimates, I conclude that for $k \geqslant n$, the integral of $\mu$ from $s_{k}-R^{\prime}$ to $s_{k+1}-R^{\prime}$ is at least $\delta$; hence any indefinite integral of $\mu$ is unbounded. This shows that $M$ satisfies Eberlein's criterion for the Anosov property.

I shall show next that focal points do not occur on $M$, under the stronger assumption

$$
b \tan 2 b r_{3}<\tanh R^{\prime},
$$

where $2 b r_{3}<\pi / 2$ (and requiring the sectional curvatures outside $B_{r_{3}}$ to be only nonpositive). The proof is highly analogous to the proof of nonconjugacy given above. I need to show that if $u$ is the solution of $u^{\prime \prime}+f u=0$ satisfying $u\left(s^{*}\right)=$ $0, u^{\prime}\left(s^{*}\right)=1$, then $u^{\prime}(s)>0$ for all $s \geqslant s^{*}$. Writing $\mu=u^{\prime} / u$, one may show that: if $\mu\left(s_{k}\right)>b \tan 2 b r_{3}$ then $\mu\left(t_{k}\right)>0$; if $\mu\left(t_{k}\right)>0$ then $\mu\left(s_{k+1}-R^{\prime}\right)>0$; and if $\mu\left(s_{k+1}-R^{\prime}\right)>0$ then $\mu\left(s_{k+1}\right)>\tanh R^{\prime}>b \tan 2 b r_{3}$. Moreover, $\mu$ is positive in each case on the interval in question. Using the behavior of $\mu$ near $s^{*}$, one may show inductively that $\mu(s)>0$, and hence $u^{\prime}(s)>0$, for all $s>s^{*}$.

Observe that it is enough to require $b \tan 2 b r_{3}<\tanh 2 R^{\prime}$ to prove nonfocality in the special case $c=1$. This includes the case that $M^{\prime}$ has constant sec- 
tional curvature -1 , as in the constructions given below. An analogous condition on the sectional curvatures along a geodesic in a surface (instability condition) was studied by E. Hopf [6, p. 592]; however, Hopf was unable to prove the existence of a surface with gaussian curvature of both signs, all of whose geodesics satisfied this condition.

The above results lead to

THEOREM 3. Suppose $\left(M^{\prime}, g^{\prime}\right)$ is a riemannian manifold, $p^{\prime} \in M^{\prime}$, such that the ball $D$ of radius $R$ centered at $p^{\prime}$ is the injective image of the corresponding ball in the tangent space at $p^{\prime}$ under the exponential map. Assume that the $g^{\prime}$ sectional curvatures are everywhere $\leqslant-c^{2}$, and have the constant value $-\beta^{2}$ on $D$, where $c \geqslant 0$ and $\beta>0$. Then there is another riemannian metric $g$ on $M^{\prime}$, $g=g^{\prime}$ except on a compact subset of $D$, which may be chosen to have any or all of the following four properties:

(1) the g-sectional curvatures are a positive constant $b^{2}$ on a neighborhood of $p^{\prime}$, and $\leqslant b^{2}$ everywhere;

(2) $g$ has no conjugate points;

(3) the geodesic flow of $g$ is Anosov, provided that $M^{\prime}$ is compact and $c>$ 0 ; and

(4) either (4A) $g$ has no focal points; or (4B) focal points occur along a certain geodesic through $p^{\prime}$, provided that $\beta R \geqslant 1.70$.

Properties (1), (2), and (3) follow from the construction given above, with no generality lost by the normalization $\beta=1$.

To obtain (4A), I fix $b$ and choose $r_{1}$ and $\epsilon$ small enough that inequality (9) is satisfied, where $r_{3}=r_{1}+\epsilon$ and $R^{\prime}=R+r_{2}-r_{3}$. Since $r_{2}$ is defined by $b^{-1} \sin b r_{1}=\sinh \left(r_{1}-r_{2}\right), r_{1}-r_{2}$ tends to zero as $r_{1}$ tends to zero; in particular, $R^{\prime}$ is bounded away from zero, so that (9) holds for small values of $r_{1}$ and $\epsilon$.

For (4B), I require the general inequality (8) and $b\left(r_{1}-\epsilon\right)>\pi / 4$. This implies that $\tanh R^{\prime}>b \tan b r_{3}>b$ and that $b \sinh \left(r_{1}-r_{2}\right)=\sin b r_{1}>\sqrt{2} / 2$; thus

$$
R>R^{\prime}+r_{1}-r_{2}>\tanh ^{-1} b+\sinh ^{-1}(1 / b \sqrt{2})=R_{0}(b) .
$$

On the other hand, if $\epsilon$ is chosen sufficiently small and $r_{1}$ sufficiently close to $\pi / 4 b$, then $R$ may be taken arbitrarily close to the value $R_{0}(b)$ defined above. Now $R_{0}(b)$ assumes a minimum value $<1.70$ at $b=0.55$; with this choice of $b$, I may thereby attain (4B). The radius 1.70 could be improved by considering a situation in which $f$ takes on more than one positive value.

Observe that a result similar to Theorem 3 may be proved by the same methods, in which a "blister" is put in the middle of each of a disjoint family of balls, where each has constant negative sectional curvature. 
Note that noncompact examples of types $A$ and $B^{2}$ with Anosov geodesic flow may be obtained by considering the universal riemannian covering manifold of $A_{0}$ and $B_{0}^{2}$, respectively.

In order to construct the compact example $A_{0}$, I now need only to find a compact manifold $M^{\prime}$ of nonpositive sectional curvature, such that the sectional curvatures are identically -1 on a nonempty open set. Theorem 3 then applies to show the existence of a manifold with properties (1), (2), (3) and (4A). But according to a result of Borel, compact manifolds of constant sectional curvature -1 exist in all dimensions [3].

The compact surface $B_{0}^{2}$, however, requires the existence of a surface $M^{\prime}$ and a point $p^{\prime} \in M^{\prime}$ such that the ball of radius $R \geqslant 1.70$ is injectively embedded by the exponential map at $p^{\prime}$ and has constant gaussian curvature -1 . The general result of Borel gives no estimate on $R$; however, a direct construction of surfaces with constant gaussian curvature -1 is feasible.

Lemma 4. For any $h \geqslant 2$ there is a compact surface $M^{\prime}$ of genus $h$ with constant gaussian curvature -1 , and a point $p^{\prime} \in M^{\prime}$, such that the exponential map at $p^{\prime}$ is injective on a ball of radius $R$, where

$$
\cosh R=1 / 2 \csc (\pi /(12 h-6)) \text {. }
$$

In particular, $R>1.71$.

PROOF. Let a regular polygon of $n=12 h-6$ sides be constructed in the two-dimensional disk with the complete hyperbolic metric of constant gaussian curvature -1 , whose interior angles are $2 \pi / 3$ and whose sides are geodesics of equal length. I shall identify appropriate sides of this polygon.

In order to see that the identifications may be chosen so that the result is topologically the surface $\Sigma$ of genus $h$, I first consider a graph on $\Sigma$ with one vertex and $2 h$ loops, whose complement is simply-connected. Now replace a small neighborhood of the vertex by a graph with $4 h-2$ vertices and $4 h-3$ new edges, such that exactly 3 edges meet at each vertex, the complement of the resulting graph remaining simply-connected. This new graph has $n / 2$ edges. By considering this graph as the boundary of its simply-connected complement, one may see that $\Sigma$ is the quotient of a polygon of $n$ sides.

Now let the identifications of the edges of the polygon be made in arclength preserving fashion. At each point of the boundary (since exactly 3 edges meet at each vertex), the identification may be extended to an isometry on a neighborhood. This permits a differentiable and riemannian structure to be defined on the quotient $M^{\prime}$. Finally, the radius $R$ (in the hyperbolic metric) of the disk inscribed in the polygon satisfies $\cosh R=1 / 2 \csc (\pi / n)$, as may be computed from the laws of hyperbolic trigonometry. 
The properties claimed for each of the four examples $A, B, A_{0}$ and $B_{0}^{2}$ follow from various strict inequalities on curvatures and distances. Thus, these properties will remain valid if the metric is replaced by one which is sufficiently close in the $C^{2}$ sense. In particular, a real-analytic metric may be found for each example. In the noncompact cases, this approximation may be carried out by means of convolution with an even, real-analytic function of the distance, in terms of a hyperbolic metric which agrees with the constructed metric off of a compact set.

\section{BIBLIOGRAPHY}

1. R. L. Bishop and R. J. Crittenden, Geometry of manifolds, Pure and Appl. Math., vol. 15, Academic Press, New York and London, 1964. MR 29 \#6401.

2. R. L. Bishop and B. O'Neill, Manifolds of negative curvature, Trans. Amer. Math. Soc. 145 (1969), 1-49. MR 40 \#4891.

3. A. Borel, Compact Clifford-Klein forms of symmetric spaces, Topology 2 (1963), 111-122. MR 26 \#3823.

4. P. Eberlein, Geodesic flow in certain manifolds without conjugate points, Trans. Amer. Math. Soc. 167 (1972), 151-170. MR 45 \#4453.

5. When is a geodesic flow of Anosov type? I, II, J. Differential Geometry 8 (1973), 437-463, 565-578.

6. E. Hopf, Statistik der Lösungen geodätischer Probleme vom unstabilen Typus. II, Math. Ann. 117 (1940), 590-608. MR 2, 106.

7. W. Klingenberg, Riemannian manifolds with geodesic flow of Anosov type, Ann. of Math. (2) 99 (1974), 1-13.

8. R. K. Miller and G. R. Sell, Existence, uniqueness and continuity of solutions of integral equations, Ann. Mat. Pura Appl. (4) 80 (1968), 135-152; Addendum: ibid. (4) 87 (1970), 281-286. MR 40 \#663; 43 \#7887.

DEPARTMENT OF MATHEMATICS, UNIVERSITY OF MINNESOTA, MINNEAPOLIS, MINNESOTA 55455 